\title{
Estimation of Coordinates on Geostationary Orbit by Using GNSS Signals
}

\author{
Valeriy Konin, Fedir Shyshkov, Olexiy Pogurelskiy \\ Department of Air navigation \\ National Aviation University \\ Kyiv, Ukraine \\ vkonin@mail.ru, shyshkovnau@mail.ua,pogurelskiy@gmail.com
}

\begin{abstract}
While facing different tasks in space, the necessity to create a reliable way of navigation for different manned on unmanned vehicles in space is required. The model in this work was created in MATLAB software package to analyze the availability of the navigation satellites in space, coordinates estimation and allows simulating different parameters. A way to navigate in space for a space vehicle is required for multiple purposes, like so-called "orbital service" or space debris extraction. Satellite navigation seems to be a cheap system for navigation in space, thus allowing to use existing system framework with only adding an extremely sensitive receiver.
\end{abstract}

Keywords - GNSS; navigation; geostationary orbit; off-nadir satellites, simulation

\section{INTRODUCTION}

The geostationary orbit lies, approximately, in a plane, which is parallel to Earth's Equator on height of about 36 thousand kilometers. Its main peculiarity is that it moves following the direction of Earth's rotation, effectively always covering same area, allowing rather specific services to be placed there.

Geostationary orbit houses communication satellites, broadcast satellites, weather satellites and other. As there is only one such convenient orbit, the number of 'slots' for geostationary satellites is extremely limited. Unfortunately, like any other satellites, the time of service of artificial objects on geostationary orbit is limited and require maintenance and removal. For that, a reliable navigation service is required.

Determination of the coordinates of a space vehicle is used for a number of tasks that are relevant in the present time (docking, movement to desired orbit), or that would be relevant in the near future (with the removal of large orbit space debris, servicing unmanned vehicle for geostationary satellites). Even though satellite navigation systems are most effective on the Earth's surface so far, the question arises - how effective are they when moving away from Earth's surface to geostationary orbit? As stated in interface control documents of GPS and GLONASS - reliable navigation using these systems is only available up to $3000 \mathrm{~km}$ for GPS [1] and $2000 \mathrm{~km}$ for GLONASS.

\section{MODEL DATA AND ASSUMPTIONS}

\section{A. Navigation satellite systems used in the research}

Definition of coordinates, velocity and time of satellite navigation systems in the geostationary orbit is performed in the radio navigation field, which is formed by radio signals emitted by satellites. To determine the coordinates in threedimensional space it is necessary to simultaneously receive signals from at least four satellites (the use of multiple constellations might require more satellites for position fix).

We can only use the two currently active Global navigation satellite systems (GNSS). Therefore, real GPS and GLONASS almanacs are used to simulate the situation on orbit. Each satellite has its own orbit parameters and a pseudo random noise code to identify the satellite.

\section{B. Physical restrictions of satellites}

The height of navigation satellites is about $20 \quad 000$ kilometers, which is way below the geostationary orbit. Therefore, we can only use the so-called off-nadir satellites, which broadcast their signal beyond the limb of Earth. You can see illustration of the off-nadir satellites on Fig. 1

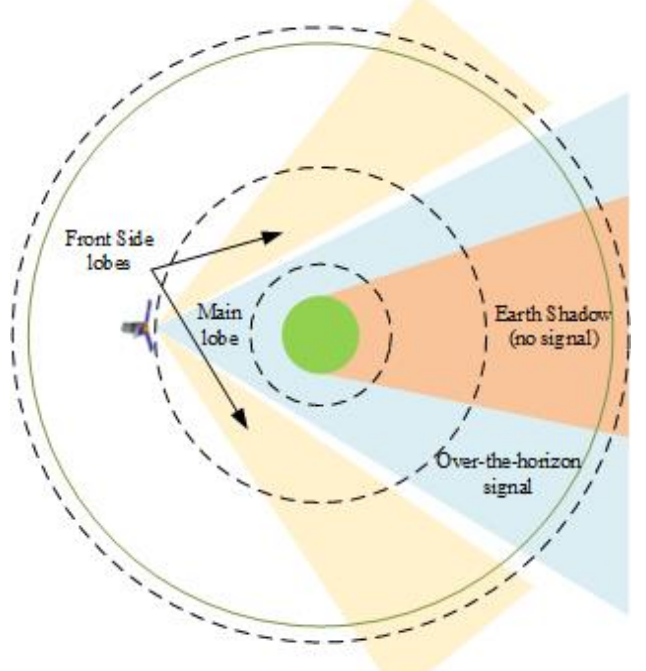

Fig. 1. The off-nadir satellite radiation 


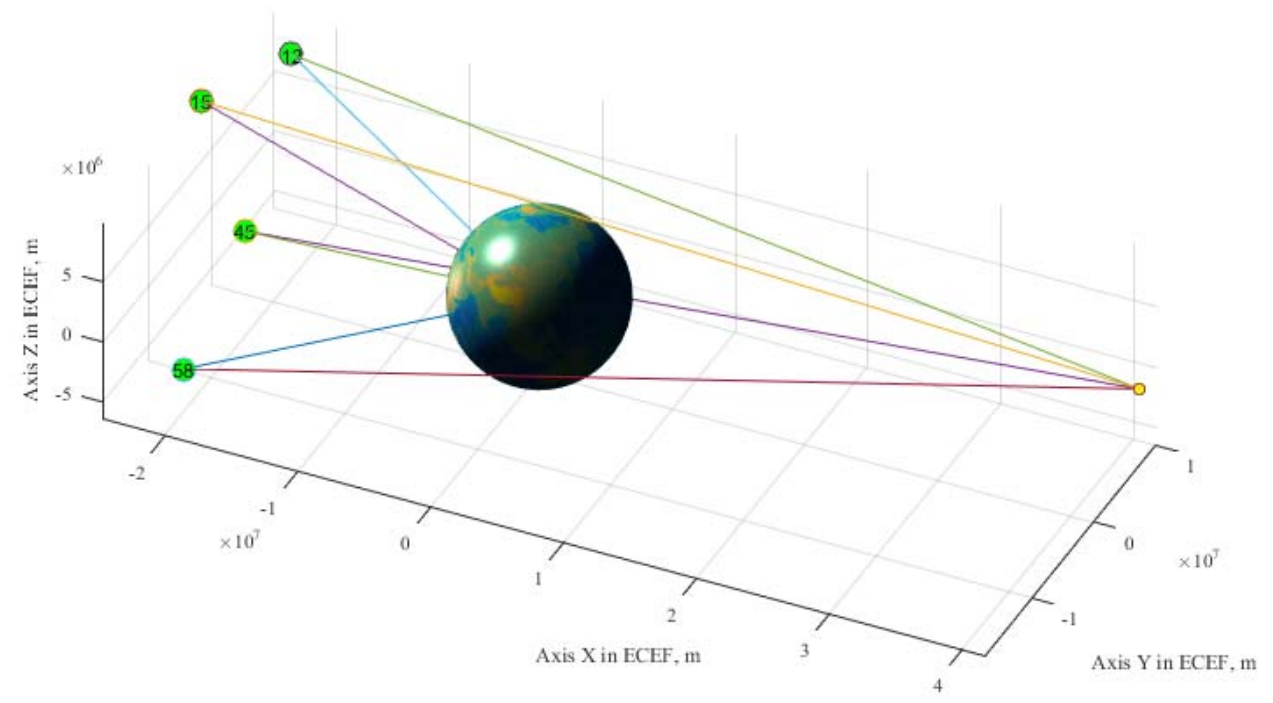

Fig. 4. Satellites visible for the best-case result

\section{CONCLUSIONS}

The satellite navigation on the geostationary orbit is only possible for limited moments of time, and even then, the possible accuracy leaves much to be desired. The use of only main lobe signals gives only limited results with low accuracy and availability.

To solve this it may be required to use the side-lobe signals of the off-nadir satellites, which allow to improve the geometrical setup of the satellites, and, in turn, the DOP factors. It will also solve the problem with the low availability of satellites, increasing the number of visible satellites for the user.

\section{REFERENCES}

[1] FAA "Global Positioning System Standard Positioning Service Performance Standard", 4th edition, September 2008, 160 p.

[2] V. Konin., A. Pogurelskiy., F. Shyshkov, "GNSS availability on geostationary orbit," 5-th international conference «Space Technologies: Present and Future», Dnepropetrovsk, 19-21 May 2015, p. 145 